\title{
Unconventional Method for Fabricating Valence Tautomeric Materials: Integrating Redox Center within a Metal-Organic Framework
}

Bao Li, ${ }^{* 1,}, 2$ Yu-Meng Zhao, ${ }^{1}$ Angelo Kirchon, ${ }^{2}$ Jian-Dong Pang, ${ }^{2}$ Xin-Yu Yang, ${ }^{2}$ Gui-Lin

Zhuang*,3 and Hong-Cai Zhou*,2,4

${ }^{1}$ Key laboratory of Material Chemistry for Energy Conversion and Storage, School of Chemistry and Chemical Engineering, Huazhong University of Science and Technology, Wuhan, Hubei 430074, People's Republic of China.

2 Department of Chemistry, Texas A\&M University, College Station, Texas 77843-3255, United States.

3 Institute of Industrial Catalysis, College of Chemical Engineering Zhejiang University of Technology, Zhejiang, 310023, People's Republic of China.

${ }^{4}$ Department of Materials Science and Engineering, Texas A\&M University, College Station, Texas 77842, United States.

*To whom correspondence should be addressed.

E-mail for B. L.: libao@hust.edu.cn; G.-L. Z.: glzhuang@zjut.edu.cn; H.-C.Z.: zhou@chem.tamu.edu 


\section{Experimental Methods}

Materials and General Methods. All starting materials were obtained commercially and were used without further purification. Elemental analyses for $\mathrm{C}, \mathrm{H}, \mathrm{N}$ were performed on a Perkin-Elmer 240Q elemental analyzer. The IR spectra were recorded in range of $400-4000 \mathrm{~cm}^{-1}$ on a Nicolet 5DX spectrometer (KBr pellets). ESR spectra were performed with EMX-10/12 spectrometer purchased from BRUKER, and the low-temperature was controlled by ER4131VT system using the liquid nitrogen. Magnetic susceptibility measurements were carried out in the temperature range of 2 $300 \mathrm{~K}$ with a magnetic field of 5000 Oe on Quantum Design MPMS XL-7 magnetometer. The tetrapyridinate ligand was synthesized by a literature method (Angew. Chem. Int. Ed. 2005, 44, 2115).

Synthesis of $\left[\mathrm{Co}_{2}(\mathrm{OAc})(3,5-\mathrm{DBcat})_{2}(\mathrm{~L})\right]$ (VT-MOF-1): To an $\mathrm{CH}_{2} \mathrm{Cl}_{2}$ /isopropanol ( 2/3 mL ) solution of L ( $70 \mathrm{mg}$ ) and 3,5-di-tert-butyl-1,2-catechol ( $88 \mathrm{mg}$ ) was layered $3 \mathrm{~mL}$ DMF. An methanol/isopropanol ( $3 / 6 \mathrm{~mL}$ ) solution of $\mathrm{Co}(\mathrm{OAc})_{2}$ ( 52 mg ) was then layered. The tube was sealed and stand at room temperature. Single crystals suitable for X-ray diffraction analysis were obtained in 2 weeks. IR ( $\mathrm{cm}^{-1}$, $\mathrm{KBr}$ ) : 3431, 2953, 1660, 1611, 1477, 1411, 1216, 1022, 828. EA results for hexane@VT-MOF-1: C, 61.87\%; H, 8.11\%; N, 6.52\%. The full formula of fresh sample should be fixed as $\left[\mathrm{Co}_{2}(\mathrm{OAc})(3,5-\mathrm{DBcat})_{2}(\mathrm{~L})\right] \cdot(\mathrm{DMF})_{3} \cdot\left(\mathrm{H}_{2} \mathrm{O}\right)_{12}$ along with the results of TGA.

Synthesis of different solvents-loaded samples: The pristine crystal samples ( 20 $\mathrm{mg}$ ) had been directly immersed the pure solvent $(20 \mathrm{~mL})$ in the vial, and the mixture is placed at room temperature. The solvent was exchanged with pure solvent every 8 hours. After three days, the solid sample had been filtered, which were utilized to measure the corresponding properties. EA results for hexane@VT-MOF-1: C, 79.64\%; H, 8.79\%; N, 3.55\%. EA results for isopropanol@VT-MOF-1: C, 71.42\%; H, 9.45\%; N, 3.38\%. EA results for tert-butanol@VT-MOF-1: C, 72.33\%; H, 9.17\%; 
N, 3.46\%. EA results for $n$-butanol@VT-MOF-1: C, 70.74\%; H, 10.42\%; N, 2.96\%.

X-Ray Structural Determination. Diffraction data for VT-MOF-1 $(0.1 \times 0.05 \times$ $0.05 \mathrm{~mm})$ was collected via Bruker Venture using $\mathrm{Cu}-K \alpha(\lambda=1.54178 \AA)$ radiation at $100 \mathrm{~K}, 200 \mathrm{~K}$ and $250 \mathrm{~K}$. The structures of complexes were solved by direct methods, and the non-hydrogen atoms were located from the trial structure and then refined anisotropically with SHELXTL using a full-matrix leastsquares procedure based on $F^{2}$ values. The hydrogen atom positions were fixed geometrically at calculated distances and allowed to ride on the parent atoms. Attempts to define the highly disordered solvent molecules were unsuccessful, so the structure was refined with the PLATON "SQUEEZE" procedure. The diffraction intensity of crystal sample was very weak due to the very small size and the nature of electrical delocalization for valence tautomeric system, which must be responsible for the corresponding alert A. The diffraction intensity of crystal sample is very weak, especially at high temperature regions. The size of crystal sample is very small, and the structure possesses large porous structure. Therefore, the collection of fully satisfied crystal data at high temperature regions is very difficult, and the resolution had been set as 1.6 A. Several sets of crystal data at $250 \mathrm{~K}$ had been collected, and the best one had been presented in this article. In addition, the disorder of hydrogen atoms on tert-butyl at high temperature must be responsible for the corresponding alert A "Maximum Shift/Error". CCDC-1842601-1842603 for the data under different temperature contain the supplementary crystallographic data for this paper. These data can be obtained free of charge from The Cambridge Crystallographic Data Centre via http://www.ccdc.cam.ac.uk/datarequest/cif.

Computational details. Spin-polarized density functional theory calculations were performed by using of Gaussian 09 software ${ }^{[1]}$. Exchange-correlation (XC) effects were treated by OPBE function for geometrical optimization and modified hybrid functional B3LYP*[2] for single-point energy calculation, which is different from standard B3LYP by reducing Hartree-Fock term of the exchange part from 0.20 
to 0.15 . Moreover, K. Yoshizawa et.al ${ }^{[3]}$ demonstrated that both OPBE and B3LYP* are better to describe the spin states of Cobalt complexes in the process of valence tautomerism. Electronic wave function of $\mathrm{C}, \mathrm{H}, \mathrm{O}, \mathrm{N}$ and $\mathrm{Co}$ were described by $6-311++\mathrm{G}^{* *}$ basic set. The initial structures were derived from the effective moieties of single-crystal diffraction results in $100 \mathrm{~K}$ and $250 \mathrm{~K}$, where the dangling bonds were saturated by hydrogen atoms. Subsequently, all atoms were optimized without any constraint and validated by frequency analysis. Moreover, in the broken symmetry ${ }^{[4]}$ calculation, fragment technique was employed in the setting of initial spin states and meanwhile corresponding wave functions were further checked by stability test. Furthermore, Mulliken ${ }^{[5]}$ spin density analysis was also carried out. The spin density and coordinate of relaxed structures were presented as following Table S4-5. In addition, according to the formula ( see EqS1 )reported by Bencini for mutinuclear complexes ${ }^{[6]}$ and Hamiltonian operator ( see EqS2 ), the relation between $J$ and energy was presented as follow:

$$
\begin{aligned}
& \Delta E\left(S-S_{\max }\right)=\sum_{i<j} J_{i j}\left(2 S_{i} S_{j}+S_{j}\right) \\
& H=-2 J_{1}\left(S_{\text {Co1 }} S_{\text {Radical }}+S_{C 02} S_{\text {Radical }}\right)-2 J_{2} S_{C 01} S_{C O 2}
\end{aligned}
$$

Where $S_{\mathrm{i}}$ is larger than $S_{j}$ in hetero-pair magnetic ions. Both $S_{\mathrm{i}}$ and $S_{\mathrm{j}}$ are one paired local spin moment, which is different from that in $\mathrm{S}_{\max }$ distribution. $\Delta \mathrm{E}\left(S-S_{\max }\right)$ is energy difference between $\mathrm{S}$ and $S_{\max }$ magnetic states. The obtained energy difference of different spin states were presented in Table S9.

Table S1. Crystal data of VT-MOF-1 at different temperature

\begin{tabular}{|c|c|c|c|}
\hline & $\mathbf{1 0 0 K}$ & $\mathbf{2 0 0 K}$ & $\mathbf{2 5 0 K}$ \\
\hline Empirical formula & $\mathrm{C}_{68} \mathrm{H}_{77} \mathrm{Co}_{2} \mathrm{~N}_{4} \mathrm{O}_{6}$ & $\mathrm{C}_{68} \mathrm{H}_{77} \mathrm{Co}_{2} \mathrm{~N}_{4} \mathrm{O}_{6}$ & $\mathrm{C}_{68} \mathrm{H}_{77} \mathrm{Co}_{2} \mathrm{~N}_{4} \mathrm{O}_{6}$ \\
\hline Formula weight & 1164.20 & 1164.20 & 1164.20 \\
\hline Temperature/K & 100.0 & 200.0 & 250.0 \\
\hline
\end{tabular}




\begin{tabular}{|c|c|c|c|}
\hline Crystal system & tetragonal & tetragonal & tetragonal \\
\hline Space group & $I-4 \mathrm{c} 2$ & $I-4 \mathrm{c} 2$ & $I-4 \mathrm{c} 2$ \\
\hline $\mathrm{a} / \AA$ & $30.049(2)$ & $30.630(2)$ & $31.034(2)$ \\
\hline $\mathrm{b} / \AA$ & $30.049(2)$ & $30.630(2)$ & $31.034(2)$ \\
\hline $\mathrm{c} / \AA ̊$ & $53.137(4)$ & $53.381(4)$ & $53.217(4)$ \\
\hline$\alpha /{ }^{\circ}$ & 90 & 90 & 90 \\
\hline$\beta /{ }^{\circ}$ & 90 & 90 & 90 \\
\hline$\gamma /{ }^{\circ}$ & 90 & 90 & 90 \\
\hline Volume $/ \AA^{3}$ & $47980(8)$ & $50081(8)$ & $51254(8)$ \\
\hline $\mathrm{Z}$ & 16 & 16 & 16 \\
\hline$\rho_{\text {calc }} \mathrm{g} / \mathrm{cm}^{3}$ & 0.638 & 0.618 & 0.604 \\
\hline$\mu / \mathrm{mm}^{-1}$ & 2.383 & 2.284 & 2.231 \\
\hline $\mathrm{F}(000)$ & 9648.0 & 9840.0 & 9840.0 \\
\hline Crystal size $/ \mathrm{mm}^{3}$ & $0.1 \times 0.05 \times 0.05$ & $0.1 \times 0.05 \times 0.05$ & $0.1 \times 0.05 \times 0.05$ \\
\hline Radiation & $\mathrm{Cu} K \alpha(\lambda=1.54178)$ & $\mathrm{Cu} K \alpha(\lambda=1.54178)$ & $\mathrm{Cu} K \alpha(\lambda=1.54178)$ \\
\hline Reflections collected & 10591 & 7667 & 3095 \\
\hline Goodness-of-fit on $\mathrm{F}^{2}$ & 0.963 & 0.915 & 0.841 \\
\hline $\begin{array}{l}\text { Final } \mathrm{R} \text { indexes } \\
\qquad[\mathrm{I}>=2 \sigma(\mathrm{I})]\end{array}$ & $\begin{array}{c}\mathrm{R}_{1}=0.0992 \\
\mathrm{wR}_{2}=0.2509\end{array}$ & $\begin{array}{c}\mathrm{R}_{1}=0.1107 \\
\mathrm{wR}_{2}=0.2516\end{array}$ & $\begin{array}{l}\mathrm{R}_{1}=0.1127 \\
\mathrm{wR}_{2}=0.2529\end{array}$ \\
\hline $\begin{array}{l}\text { Final } \mathrm{R} \text { indexes [all } \\
\text { data] }\end{array}$ & $\begin{array}{l}\mathrm{R}_{1}=0.1487 \\
\mathrm{wR}_{2}=0.2712\end{array}$ & $\begin{array}{l}\mathrm{R}_{1}=0.2315 \\
\mathrm{wR}_{2}=0.3051\end{array}$ & $\begin{array}{l}\mathrm{R}_{1}=0.2233 \\
\mathrm{wR}_{2}=0.3129\end{array}$ \\
\hline
\end{tabular}


Table S2. Selected bond lengths of VT-MOF-1 at different temperature

\begin{tabular}{cccc}
\hline & $\mathbf{1 0 0 k}$ & $\mathbf{2 0 0} \mathbf{K}$ & $\mathbf{2 5 0 k}$ \\
\hline $\mathrm{Co}(1)-\mathrm{O}(1)$ & $1.996(1)$ & $2.138(1)$ & $2.223(1)$ \\
$\mathrm{Co}(1)-\mathrm{O}(3)$ & $1.957(2)$ & $2.006(2)$ & $2.109(2)$ \\
$\mathrm{Co}(1)-\mathrm{O}(4)$ & $1.883(1)$ & $2.027(1)$ & $2.086(1)$ \\
$\mathrm{Co}(1)-\mathrm{O}(6)$ & $1.929(1)$ & $1.994(1)$ & $1.957(1)$ \\
$\mathrm{Co}(1)-\mathrm{N}(2)$ & $1.994(1)$ & $2.110(1)$ & $2.073(1)$ \\
$\mathrm{Co}(1)-\mathrm{N}(3)$ & $1.962(1)$ & $2.076(1)$ & $2.067(2)$ \\
\hline
\end{tabular}

\begin{tabular}{rrrr}
\hline $\mathrm{C}(53)-\mathrm{O}(3)$ & $1.368(2)$ & $1.293(2)$ & $1.391(1)$ \\
$\mathrm{C}(54)-\mathrm{O}(4)$ & $1.358(1)$ & $1.333(1)$ & $1.317(1)$ \\
$\mathrm{C}(53)-\mathrm{C}(54)$ & $1.378(1)$ & $1.458(1)$ & $1.453(2)$ \\
\hline
\end{tabular}

\begin{tabular}{llll}
\hline $\mathrm{Co}(2)-\mathrm{O}(1)$ & $1.968(2)$ & $2.092(2)$ & $2.026(2)$ \\
$\mathrm{Co}(2)-\mathrm{O}(2)$ & $1.814(1)$ & $2.053(1)$ & $2.164(1)$ \\
$\mathrm{Co}(2)-\mathrm{O}(3)$ & $1.959(1)$ & $1.993(1)$ & $2.003(1)$ \\
$\mathrm{Co}(2)-\mathrm{O}(5)$ & $1.999(1)$ & $2.003(1)$ & $2.139(1)$ \\
$\mathrm{Co}(2)-\mathrm{N}(1)$ & $1.952(1)$ & $2.008(1)$ & $2.111(1)$ \\
$\mathrm{Co}(2)-\mathrm{N}(4)$ & $2.017(2)$ & $2.107(2)$ & $2.124(2)$ \\
\hline
\end{tabular}

$\begin{array}{llll}\mathrm{C}(39)-\mathrm{O}(1) & 1.329(1) & 1.348(1) & 1.380(1) \\ \mathrm{C}(40)-\mathrm{O}(2) & 1.327(1) & 1.347(1) & 1.370(1)\end{array}$




$\mathrm{C}(39)-\mathrm{C}(40) \quad 1.385(1) \quad 1.417(1) \quad 1.413(1)$

Table S3. The calculation of bond valence sum for Co1 and Co2 atoms of VT-MOF-1 at different temperature

\begin{tabular}{lcccc}
\hline & \multicolumn{2}{c}{$\mathrm{Co}(1)$} & \multicolumn{2}{c}{$\mathrm{Co}(2)$} \\
\cline { 2 - 5 } & $\mathrm{Co}(\mathrm{II})$ & $\mathrm{Co}(\mathrm{III})$ & $\mathrm{Co}(\mathrm{II})$ & $\mathrm{Co}(\mathrm{III})$ \\
\hline $100 \mathrm{~K}$ & 2.976 & 3.041 & 3.026 & 3.092 \\
\hline $200 \mathrm{~K}$ & 2.251 & 2.301 & 2.342 & 2.394 \\
\hline $250 \mathrm{~K}$ & 2.115 & 2.162 & 2.048 & 2.093 \\
\hline
\end{tabular}

Table S4 . The collection of spin density of cobalt centers and two catechol units in the state of $S=3 / 2$ and $S=2 \times(3 / 2)+1 / 2$.

\begin{tabular}{|c|c|c|c|c|c|c|c|}
\hline \multicolumn{4}{|c|}{$S=3 / 2$} & \multicolumn{4}{|c|}{$S=2 \times(3 / 2)+1 / 2$} \\
\hline Col & 0.049119 & $\mathrm{C}$ & -0.013069 & Col & 2.531977 & $\mathrm{C}$ & -0.001765 \\
\hline $\mathrm{Co} 2$ & 2.48234 & $\mathrm{C}$ & 0.001985 & $\mathrm{Co} 2$ & 2.532386 & $\mathrm{H}$ & 0.001037 \\
\hline $\mathrm{O} 1$ & 0.034494 & $\mathrm{H}$ & 0.000162 & O1 & 0.205028 & $\mathrm{C}$ & 0.012234 \\
\hline $\mathrm{O} 2$ & -0.00238 & $\mathrm{C}$ & 0.000038 & $\mathrm{O} 2$ & 0.226095 & $\mathrm{C}$ & 0.000725 \\
\hline $\mathrm{O} 3$ & 0.027084 & $\mathrm{C}$ & 0.006907 & $\mathrm{O} 3$ & 0.204584 & $\mathrm{H}$ & 0.000715 \\
\hline $\mathrm{O} 4$ & 0.128369 & $\mathrm{C}$ & -0.005587 & $\mathrm{O} 4$ & 0.225368 & $\mathrm{C}$ & -0.007325 \\
\hline $\mathrm{O} 5$ & -0.00297 & $\mathrm{H}$ & 0.002368 & O5 & 0.048917 & $\mathrm{H}$ & 0.003078 \\
\hline $\mathrm{O} 6$ & 0.037969 & $\mathrm{C}$ & -0.01509 & O6 & 0.049826 & $\mathrm{C}$ & -0.036724 \\
\hline C & -0.0063 & $\mathrm{H}$ & 0.000239 & $\mathrm{C}$ & -0.00682 & $\mathrm{H}$ & -0.000587 \\
\hline
\end{tabular}




\begin{tabular}{|c|c|c|c|c|c|c|c|}
\hline $\mathrm{H}$ & -0.00059 & $\mathrm{C}$ & -0.006811 & $\mathrm{H}$ & 0.002925 & $\mathrm{C}$ & 0.047372 \\
\hline $\mathrm{C}$ & 0.007087 & $\mathrm{C}$ & 0.005993 & $\mathrm{C}$ & 0.067136 & $\mathrm{C}$ & -0.007512 \\
\hline $\mathrm{C}$ & 0.026663 & $\mathrm{H}$ & 0.000711 & $\mathrm{C}$ & 0.146932 & $\mathrm{C}$ & 0.005361 \\
\hline $\mathrm{C}$ & 0.002231 & $\mathrm{C}$ & -0.008672 & $\mathrm{C}$ & -0.03262 & $\mathrm{H}$ & 0.000819 \\
\hline $\mathrm{H}$ & $-9.4 \mathrm{E}-05$ & $\mathrm{H}$ & 0.002736 & $\mathrm{H}$ & -0.00063 & $\mathrm{C}$ & -0.014551 \\
\hline $\mathrm{C}$ & 0.001288 & $\mathrm{~N}$ & 0.071375 & $\mathrm{C}$ & $-3.8 \mathrm{E}-05$ & $\mathrm{H}$ & -0.003529 \\
\hline $\mathrm{H}$ & 0.000043 & $\mathrm{C}$ & -0.004202 & $\mathrm{H}$ & 0.000767 & $\mathrm{~N}$ & 0.073899 \\
\hline $\mathrm{C}$ & 0.000145 & $\mathrm{H}$ & 0.001919 & $\mathrm{C}$ & -0.01132 & $\mathrm{C}$ & -0.002653 \\
\hline $\mathrm{C}$ & 0.006841 & $\mathrm{C}$ & 0.003923 & $\mathrm{C}$ & 0.053026 & $\mathrm{H}$ & 0.002162 \\
\hline $\mathrm{H}$ & -0.00052 & $\mathrm{H}$ & 0.000597 & $\mathrm{H}$ & -0.00258 & $\mathrm{C}$ & 0.009012 \\
\hline $\mathrm{C}$ & 0.020174 & $\mathrm{C}$ & 0.000398 & $\mathrm{C}$ & 0.013675 & $\mathrm{H}$ & 0.00071 \\
\hline $\mathrm{C}$ & -0.00463 & $\mathrm{C}$ & -0.000495 & $\mathrm{C}$ & 0.065881 & $\mathrm{C}$ & 0.00645 \\
\hline $\mathrm{C}$ & -0.0125 & $\mathrm{C}$ & -0.000276 & $\mathrm{C}$ & 0.053455 & $\mathrm{H}$ & 0.000817 \\
\hline $\mathrm{H}$ & 0.001103 & $\mathrm{H}$ & -0.000139 & $\mathrm{H}$ & -0.00253 & $\mathrm{C}$ & -0.007827 \\
\hline $\mathrm{C}$ & 0.029402 & $\mathrm{C}$ & 0.000784 & $\mathrm{C}$ & 0.154652 & $\mathrm{C}$ & -0.013108 \\
\hline $\mathrm{C}$ & 0.000662 & $\mathrm{H}$ & 0.000011 & $\mathrm{C}$ & 0.009176 & $\mathrm{H}$ & -0.003355 \\
\hline $\mathrm{H}$ & 0.000769 & $\mathrm{~N}$ & 0.001816 & $\mathrm{H}$ & 0.000517 & $\mathrm{C}$ & 0.009109 \\
\hline $\mathrm{C}$ & -0.00872 & $\mathrm{C}$ & 0.000837 & $\mathrm{C}$ & -0.0037 & $\mathrm{H}$ & 0.000759 \\
\hline $\mathrm{H}$ & 0.002679 & $\mathrm{H}$ & 0.000169 & $\mathrm{H}$ & 0.001034 & $\mathrm{~N}$ & 0.073555 \\
\hline $\mathrm{N}$ & 0.081166 & $\mathrm{H}$ & 0.000132 & $\mathrm{~N}$ & 0.076038 & $\mathrm{C}$ & -0.004425 \\
\hline $\mathrm{C}$ & 0.001017 & $\mathrm{H}$ & 0.000658 & $\mathrm{C}$ & 0.008843 & $\mathrm{H}$ & 0.002083 \\
\hline $\mathrm{H}$ & $-2.2 \mathrm{E}-05$ & $\mathrm{H}$ & 0.000698 & $\mathrm{H}$ & 0.000542 & $\mathrm{H}$ & 0.000457 \\
\hline $\mathrm{C}$ & -0.00505 & $\mathrm{H}$ & -0.000007 & $\mathrm{C}$ & 0.080862 & $\mathrm{H}$ & -0.005025 \\
\hline $\mathrm{C}$ & 0.011137 & $\mathrm{H}$ & 0.000029 & $\mathrm{C}$ & 0.021701 & $\mathrm{H}$ & -0.003571 \\
\hline
\end{tabular}




\begin{tabular}{llllllll}
\hline $\mathrm{H}$ & 0.000149 & $\mathrm{H}$ & -0.000011 & $\mathrm{H}$ & 0.000564 & $\mathrm{H}$ & 0.000454 \\
$\mathrm{H}$ & 0.000203 & $\mathrm{H}$ & -0.001498 & $\mathrm{H}$ & 0.00065 & $\mathrm{H}$ & -0.003565 \\
$\mathrm{H}$ & 0.001303 & $\mathrm{H}$ & -0.001168 & $\mathrm{H}$ & 0.001383 & $\mathrm{H}$ & -0.004965 \\
$\mathrm{C}$ & 0.023169 & $\mathrm{H}$ & 0.000275 & $\mathrm{C}$ & 0.080344 & $\mathrm{H}$ & 0.000195 \\
$\mathrm{C}$ & 0.020453 & $\mathrm{H}$ & 0.000316 & $\mathrm{~N}$ & 0.076322 & $\mathrm{H}$ & 0.000199 \\
$\mathrm{~N}$ & -0.00133 & & & $\mathrm{C}$ & -0.01108 & & \\
\hline
\end{tabular}

Table S5 . The collection of spin density of cobalt centers and two catechol units in the state of $S=2 \times(3 / 2)+1 / 2$.

\begin{tabular}{cccc}
\hline \multicolumn{2}{c}{ Catechol chelated on Co1 } & \multicolumn{2}{c}{ Catechol chelated on Co2 } \\
\hline $\mathrm{O} 1$ & 0.205028 & $\mathrm{O} 3$ & 0.204584 \\
$\mathrm{O} 2$ & 0.226095 & $\mathrm{O} 4$ & 0.225368 \\
$\mathrm{C} 12$ & 0.146932 & $\mathrm{C} 24$ & 0.154652 \\
$\mathrm{C} 11$ & 0.067136 & $\mathrm{C} 21$ & 0.065881 \\
$\mathrm{C} 13$ & 0.080344 & $\mathrm{C} 22$ & 0.053455 \\
$\mathrm{C} 18$ & 0.053026 & $\mathrm{C} 20$ & 0.013675 \\
$\mathrm{C} 32$ & 0.080862 & $\mathrm{C} 37$ & -0.03262 \\
$\mathrm{C} 42$ & 0.012234 & $\mathrm{C} 47$ & -0.03672 \\
\hline
\end{tabular}

* $\mathrm{O} 1$ and $\mathrm{O} 2$ atoms connect to $\mathrm{C} 12$ and $\mathrm{C} 11$, repectively; $\mathrm{O} 3$ and $\mathrm{O} 4$ connect with $\mathrm{C} 24$ and $\mathrm{C} 25$, respectively.

Table S6. Relaxed structures of the spin states $\mathrm{S}=3 / 2$ and $\mathrm{S}=2 \times(3 / 2)+1 / 2$.

$$
\left[\mathrm{Co}_{2}(\mathrm{OAc})(3,5-\mathrm{DBcat})_{2}(\text { pyridine })_{4}\right] \text { with } \mathrm{S}=3 / 2
$$




\begin{tabular}{|c|c|c|c|c|c|c|c|}
\hline Co & 1.54555 & 0.021704 & -0.23337 & $\mathrm{C}$ & -4.89935 & -3.63497 & -0.36918 \\
\hline Co & -1.56189 & -0.06108 & -0.13817 & $\mathrm{C}$ & 4.033307 & 1.380293 & 0.21906 \\
\hline $\mathrm{O}$ & 0.199856 & -1.3013 & 0.087756 & $\mathrm{H}$ & 4.199398 & 0.483364 & 0.808221 \\
\hline $\mathrm{O}$ & 2.118251 & -0.27195 & 1.521003 & $\mathrm{C}$ & 1.714768 & -1.76943 & 3.363136 \\
\hline $\mathrm{O}$ & 1.050013 & 0.343942 & -2.11856 & $\mathrm{C}$ & -3.83518 & -3.60671 & -1.27016 \\
\hline $\mathrm{O}$ & 0.135992 & 1.29882 & 0.301741 & $\mathrm{C}$ & -2.90992 & -2.57206 & -1.18992 \\
\hline $\mathrm{O}$ & -1.98587 & 0.410569 & 1.693751 & $\mathrm{H}$ & -2.06654 & -2.51305 & -1.87387 \\
\hline $\mathrm{O}$ & -1.13922 & -0.20941 & -2.20069 & $\mathrm{C}$ & 0.785273 & 2.726179 & 2.147182 \\
\hline $\mathrm{C}$ & 2.54619 & 2.571523 & -1.09383 & $\mathrm{H}$ & 1.646017 & 3.100744 & 1.600194 \\
\hline $\mathrm{H}$ & 1.558528 & 2.598079 & -1.54047 & $\mathrm{C}$ & -4.91414 & 3.364782 & -1.76359 \\
\hline $\mathrm{C}$ & 1.434429 & -1.26591 & 2.084954 & $\mathrm{C}$ & -4.71167 & 2.175043 & -2.4602 \\
\hline $\mathrm{C}$ & 0.414852 & -1.86539 & 1.306545 & $\mathrm{H}$ & -5.25771 & 1.954523 & -3.37599 \\
\hline $\mathrm{C}$ & -0.25702 & -2.99414 & 1.778644 & $\mathrm{C}$ & -3.28287 & 2.599872 & -0.17683 \\
\hline $\mathrm{H}$ & -1.01474 & -3.46624 & 1.157473 & $\mathrm{H}$ & -2.70147 & 2.732541 & 0.731719 \\
\hline $\mathrm{C}$ & 3.442189 & 3.626484 & -1.21812 & $\mathrm{~N}$ & -3.08244 & 1.448866 & -0.84092 \\
\hline $\mathrm{H}$ & 3.152733 & 4.503932 & -1.79315 & $\mathrm{C}$ & -3.79132 & 1.255095 & -1.96601 \\
\hline $\mathrm{C}$ & 4.68555 & 3.544093 & -0.59405 & $\mathrm{H}$ & -3.59603 & 0.323261 & -2.49008 \\
\hline $\mathrm{C}$ & 1.004905 & -2.87284 & 3.843361 & $\mathrm{C}$ & -4.18104 & 3.577535 & -0.59798 \\
\hline $\mathrm{H}$ & 1.221662 & -3.25408 & 4.841334 & $\mathrm{H}$ & -4.29629 & 4.488302 & -0.01309 \\
\hline $\mathrm{C}$ & -1.43273 & 1.77482 & 3.564279 & $\mathrm{C}$ & 4.387689 & -1.98738 & -2.67407 \\
\hline $\mathrm{C}$ & -1.1787 & 1.265977 & 2.271956 & $\mathrm{C}$ & 4.633445 & -3.19578 & -2.02516 \\
\hline $\mathrm{C}$ & -0.5948 & 2.726003 & 4.134668 & $\mathrm{C}$ & 3.086749 & -2.48506 & -0.32805 \\
\hline $\mathrm{H}$ & -0.80271 & 3.097166 & 5.13881 & $\mathrm{H}$ & 2.550344 & -2.65608 & 0.600841 \\
\hline $\mathrm{C}$ & -0.03218 & 1.752007 & 1.550723 & $\mathrm{C}$ & 3.964455 & -3.44121 & -0.82992 \\
\hline
\end{tabular}




\begin{tabular}{|c|c|c|c|c|c|c|c|}
\hline $\mathrm{C}$ & -4.98285 & -2.61684 & 0.580765 & $\mathrm{H}$ & 4.109429 & -4.36542 & -0.2742 \\
\hline $\mathrm{H}$ & -5.78601 & -2.59302 & 1.315224 & $\mathrm{~N}$ & 2.84539 & -1.31649 & -0.95212 \\
\hline $\mathrm{C}$ & -4.01729 & -1.61626 & 0.589929 & $\mathrm{C}$ & 3.495555 & -1.08339 & -2.11071 \\
\hline $\mathrm{H}$ & -4.03049 & -0.81404 & 1.324472 & $\mathrm{H}$ & 3.265249 & -0.14255 & -2.59896 \\
\hline $\mathrm{N}$ & -2.98953 & -1.57753 & -0.28297 & $\mathrm{H}$ & -5.62649 & 4.108111 & -2.12029 \\
\hline $\mathrm{C}$ & 4.975237 & 2.399156 & 0.14561 & $\mathrm{H}$ & -5.64123 & -4.43185 & -0.40304 \\
\hline $\mathrm{H}$ & 5.922157 & 2.285406 & 0.669529 & $\mathrm{H}$ & -3.71515 & -4.37843 & -2.02883 \\
\hline $\mathrm{C}$ & 0.030815 & -3.48868 & 3.055901 & $\mathrm{H}$ & 5.327176 & -3.92523 & -2.4413 \\
\hline $\mathrm{C}$ & 0.008839 & 0.219522 & -4.23615 & $\mathrm{H}$ & 4.878987 & -1.73506 & -3.61194 \\
\hline $\mathrm{H}$ & 0.135827 & -0.7877 & -4.65439 & $\mathrm{H}$ & 5.406527 & 4.356593 & -0.67312 \\
\hline $\mathrm{H}$ & -0.93209 & 0.622549 & -4.62231 & $\mathrm{H}$ & -2.29823 & 1.387587 & 4.101306 \\
\hline $\mathrm{H}$ & 0.849208 & 0.835726 & -4.56709 & $\mathrm{H}$ & 1.164066 & 3.960586 & 3.869791 \\
\hline $\mathrm{C}$ & -0.04624 & 0.113122 & -2.72259 & $\mathrm{H}$ & -0.5127 & -4.35518 & 3.431216 \\
\hline $\mathrm{C}$ & 0.512457 & 3.208822 & 3.424664 & $\mathrm{H}$ & 2.48438 & -1.28434 & 3.962172 \\
\hline $\mathrm{N}$ & 2.837817 & 1.453157 & -0.39938 & & & & \\
\hline
\end{tabular}

\begin{tabular}{|c|c|c|c|c|c|c|c|}
\hline \multicolumn{8}{|c|}{$\left[\mathrm{Co}_{2}(\mathrm{OAc})(3,5-\mathrm{DBcat})_{2}(\text { pyridine })_{4}\right]$ with $\mathrm{S}=2 \times(3 / 2)+1 / 2$} \\
\hline Co & -1.54111 & -0.04644 & -0.13581 & $\mathrm{C}$ & -4.19408 & -1.45457 & 0.488746 \\
\hline Co & 1.546576 & 0.042935 & -0.14246 & $\mathrm{H}$ & -4.25418 & -0.58124 & 1.1342 \\
\hline $\mathrm{O}$ & -0.02755 & 1.355339 & 0.359713 & $\mathrm{C}$ & -1.78611 & 1.945381 & 3.486789 \\
\hline $\mathrm{O}$ & -2.15359 & 0.498694 & 1.612952 & $\mathrm{C}$ & 3.9037 & 3.628226 & -1.12329 \\
\hline $\mathrm{O}$ & -1.04577 & -0.40103 & -2.13562 & $\mathrm{H}$ & 3.736873 & 4.478192 & -1.78265 \\
\hline $\mathrm{O}$ & 0.032714 & -1.35833 & 0.357154 & $\mathrm{C}$ & 2.956646 & 2.611027 & -1.05826 \\
\hline $\mathrm{O}$ & 2.169157 & -0.51468 & 1.59853 & $\mathrm{H}$ & 2.049145 & 2.64007 & -1.65659 \\
\hline
\end{tabular}




\begin{tabular}{|c|c|c|c|c|c|c|c|}
\hline $\mathrm{O}$ & 1.045989 & 0.421315 & -2.13766 & $\mathrm{C}$ & -0.52021 & -2.86858 & 2.171474 \\
\hline $\mathrm{C}$ & -2.94274 & -2.61314 & -1.05925 & $\mathrm{H}$ & -1.42043 & -3.22061 & 1.673425 \\
\hline $\mathrm{H}$ & -2.03038 & -2.64268 & -1.65018 & $\mathrm{C}$ & -0.00537 & 0.024229 & -2.71391 \\
\hline $\mathrm{C}$ & -1.44013 & 1.409094 & 2.23083 & $\mathrm{C}$ & 4.579364 & -3.41207 & -2.27891 \\
\hline $\mathrm{C}$ & -0.25613 & 1.877125 & 1.558426 & $\mathrm{C}$ & 4.484737 & -2.1268 & -2.80926 \\
\hline $\mathrm{C}$ & 0.532008 & 2.859813 & 2.176247 & $\mathrm{H}$ & 5.051404 & -1.83446 & -3.69171 \\
\hline $\mathrm{H}$ & 1.426192 & 3.218506 & 1.672087 & $\mathrm{C}$ & 3.009976 & -2.72945 & -0.59655 \\
\hline $\mathrm{C}$ & -3.88964 & -3.62995 & -1.132 & $\mathrm{H}$ & 2.410676 & -2.93587 & 0.285988 \\
\hline $\mathrm{H}$ & -3.71856 & -4.47925 & -1.79111 & $\mathrm{~N}$ & 2.916275 & -1.48742 & -1.09791 \\
\hline $\mathrm{C}$ & -5.03988 & -3.53891 & -0.3496 & $\mathrm{C}$ & 3.644184 & -1.2057 & -2.19051 \\
\hline $\mathrm{C}$ & -0.98361 & 2.916083 & 4.077451 & $\mathrm{H}$ & 3.531819 & -0.19861 & -2.58362 \\
\hline $\mathrm{H}$ & -1.25099 & 3.320907 & 5.053438 & $\mathrm{C}$ & 3.823245 & -3.71613 & -1.14969 \\
\hline $\mathrm{C}$ & 1.812792 & -1.97061 & 3.467319 & $\mathrm{H}$ & 3.854784 & -4.70403 & -0.69386 \\
\hline $\mathrm{C}$ & 1.458543 & -1.42655 & 2.21705 & $\mathrm{C}$ & -4.4825 & 2.137904 & -2.79287 \\
\hline $\mathrm{C}$ & 1.011033 & -2.9408 & 4.059704 & $\mathrm{H}$ & -5.02762 & 1.857192 & -3.69252 \\
\hline $\mathrm{H}$ & 1.284765 & -3.35177 & 5.031349 & $\mathrm{C}$ & -4.61594 & 3.405439 & -2.22922 \\
\hline $\mathrm{C}$ & 0.267371 & -1.88657 & 1.551931 & $\mathrm{C}$ & -3.0593 & 2.712141 & -0.53929 \\
\hline $\mathrm{C}$ & 5.191816 & 2.424473 & 0.495116 & $\mathrm{H}$ & -2.48102 & 2.907418 & 0.359707 \\
\hline $\mathrm{H}$ & 6.060932 & 2.306357 & 1.1397 & $\mathrm{C}$ & -3.88591 & 3.695104 & -1.0792 \\
\hline $\mathrm{C}$ & 4.197815 & 1.450675 & 0.496532 & $\mathrm{H}$ & -3.94804 & 4.668926 & -0.59697 \\
\hline $\mathrm{H}$ & 4.253652 & 0.576402 & 1.141122 & $\mathrm{~N}$ & -2.92781 & 1.487088 & -1.07304 \\
\hline $\mathrm{N}$ & 3.093799 & 1.529751 & -0.26884 & $\mathrm{C}$ & -3.63077 & 1.219289 & -2.18556 \\
\hline $\mathrm{C}$ & -5.18825 & -2.42809 & 0.479493 & $\mathrm{H}$ & -3.4858 & 0.227159 & -2.60518 \\
\hline $\mathrm{H}$ & -6.06148 & -2.31081 & 1.118635 & $\mathrm{H}$ & -5.8003 & -4.31844 & -0.38093 \\
\hline
\end{tabular}




\begin{tabular}{lllllllll}
\hline $\mathrm{C}$ & 0.168879 & 3.371829 & 3.422125 & $\mathrm{H}$ & -0.77055 & -4.15201 & 3.879446 \\
$\mathrm{C}$ & -0.03867 & 0.088503 & -4.22969 & $\mathrm{H}$ & 2.715494 & -1.60333 & 3.954617 \\
$\mathrm{H}$ & -0.538 & 1.01844 & -4.53232 & $\mathrm{H}$ & 5.809058 & 4.316296 & -0.35844 \\
$\mathrm{H}$ & 0.970088 & 0.089941 & -4.65096 & $\mathrm{H}$ & -2.68309 & 1.57165 & 3.979741 \\
$\mathrm{H}$ & -0.61929 & -0.7446 & -4.63786 & $\mathrm{H}$ & 0.79081 & 4.136439 & 3.888061 \\
$\mathrm{C}$ & -0.149 & -3.38808 & 3.411901 & $\mathrm{H}$ & -5.27315 & 4.150177 & -2.67722 \\
$\mathrm{~N}$ & -3.08515 & -1.53283 & -0.26948 & $\mathrm{H}$ & 5.226876 & -4.15938 & -2.73662 \\
$\mathrm{C}$ & 5.048663 & 3.53651 & -0.33326 & & & & \\
\hline
\end{tabular}

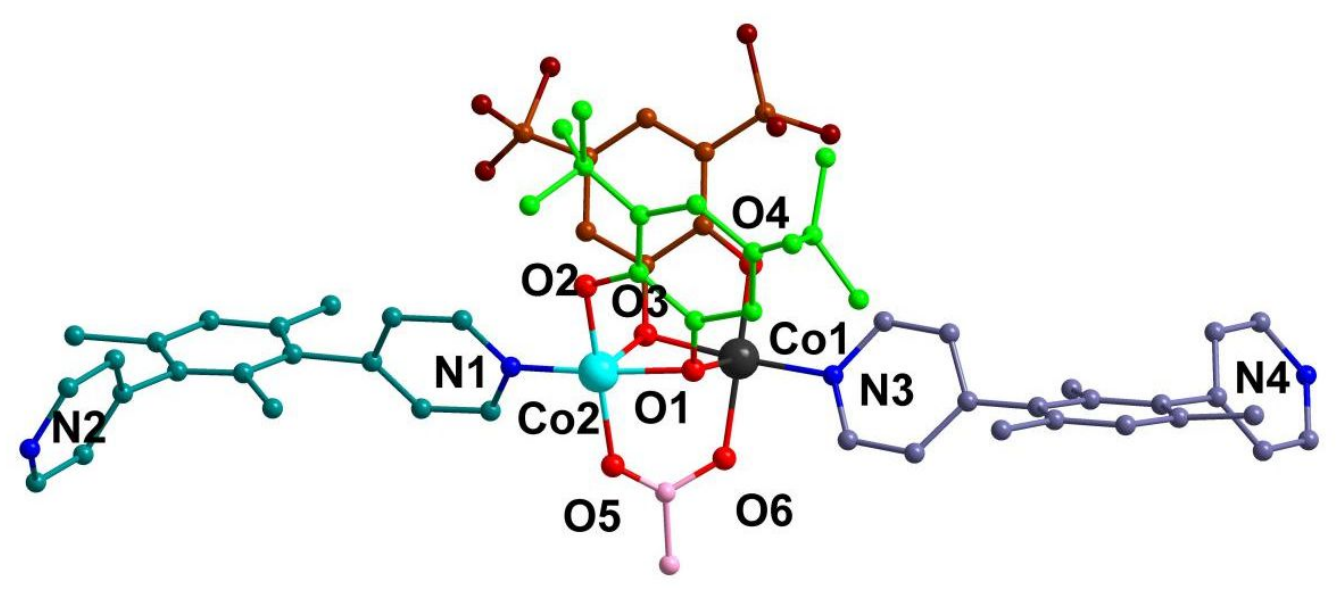




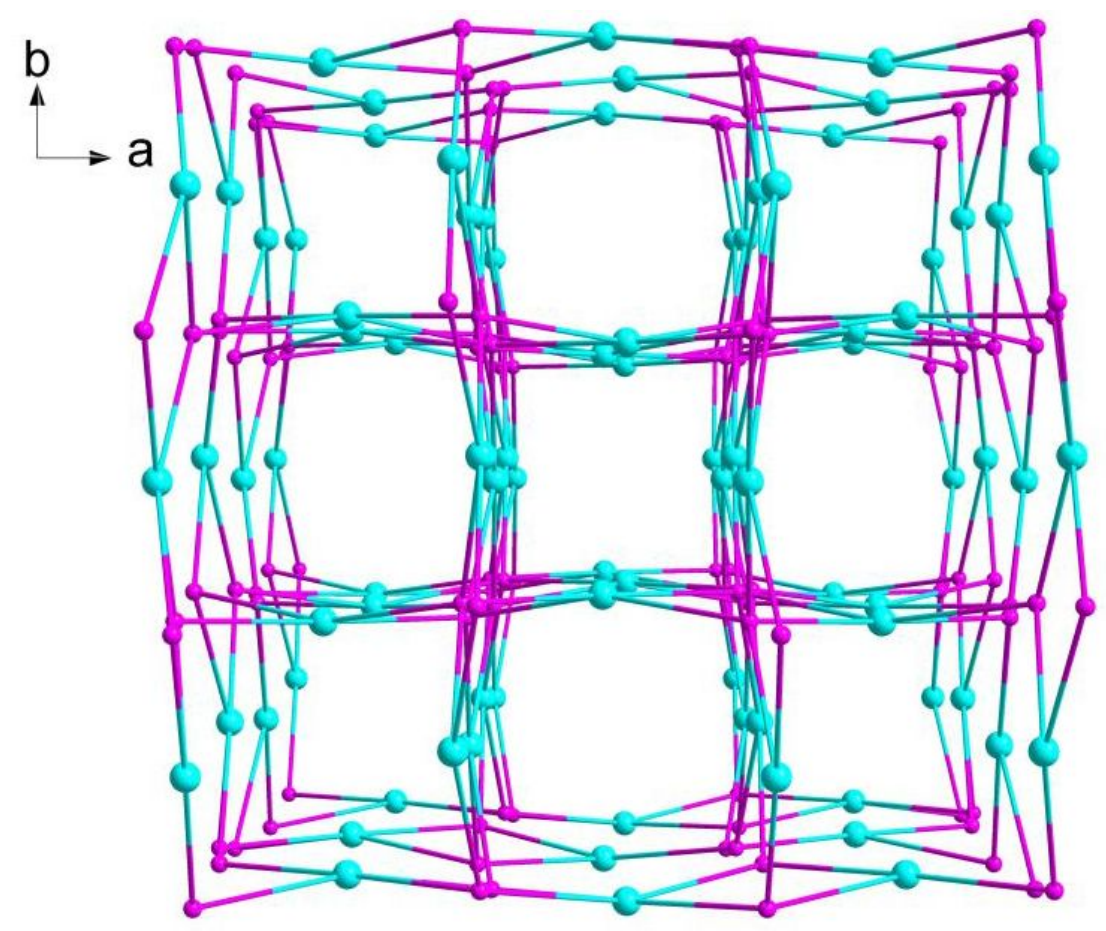

Figure S1. The perspective view of asymmetric unit and the topological structure of VT-MOF-1. Blue and purple balls represent the dinuclear clusters and tetrapyridine linkers.

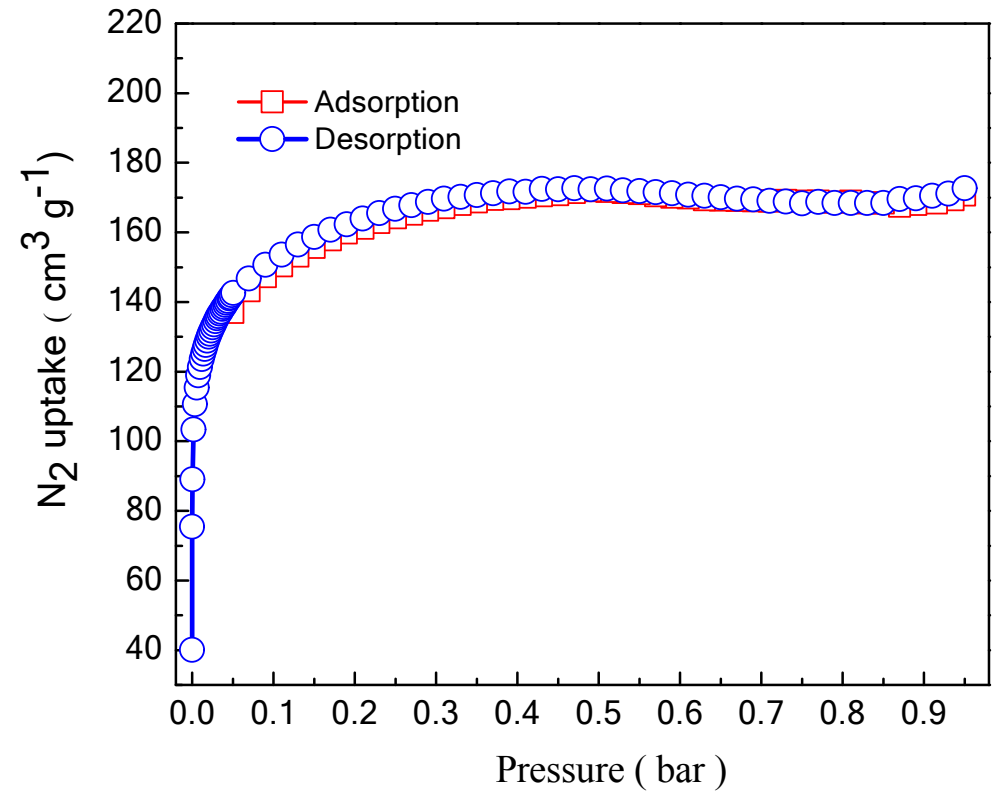


Figure S2. $\mathrm{N}_{2}$ sorption isotherms for activated sample of VT-MOF-1 at $77 \mathrm{~K}$ after vacuum-drying at $60{ }^{\circ} \mathrm{C}$ overnight. The activated sample adsorbed $173 \mathrm{~cm}^{3} \mathrm{~g}^{-1}$ of $\mathrm{N}_{2}$ at $77 \mathrm{~K}$, and the Brunauer-Emmett-Teller (BET) and Langmuir surface areas are calculated as $609 \mathrm{~m}^{2} \mathrm{~g}^{-1}$ and $932 \mathrm{~m}^{2} \mathrm{~g}^{-1}$, respectively.

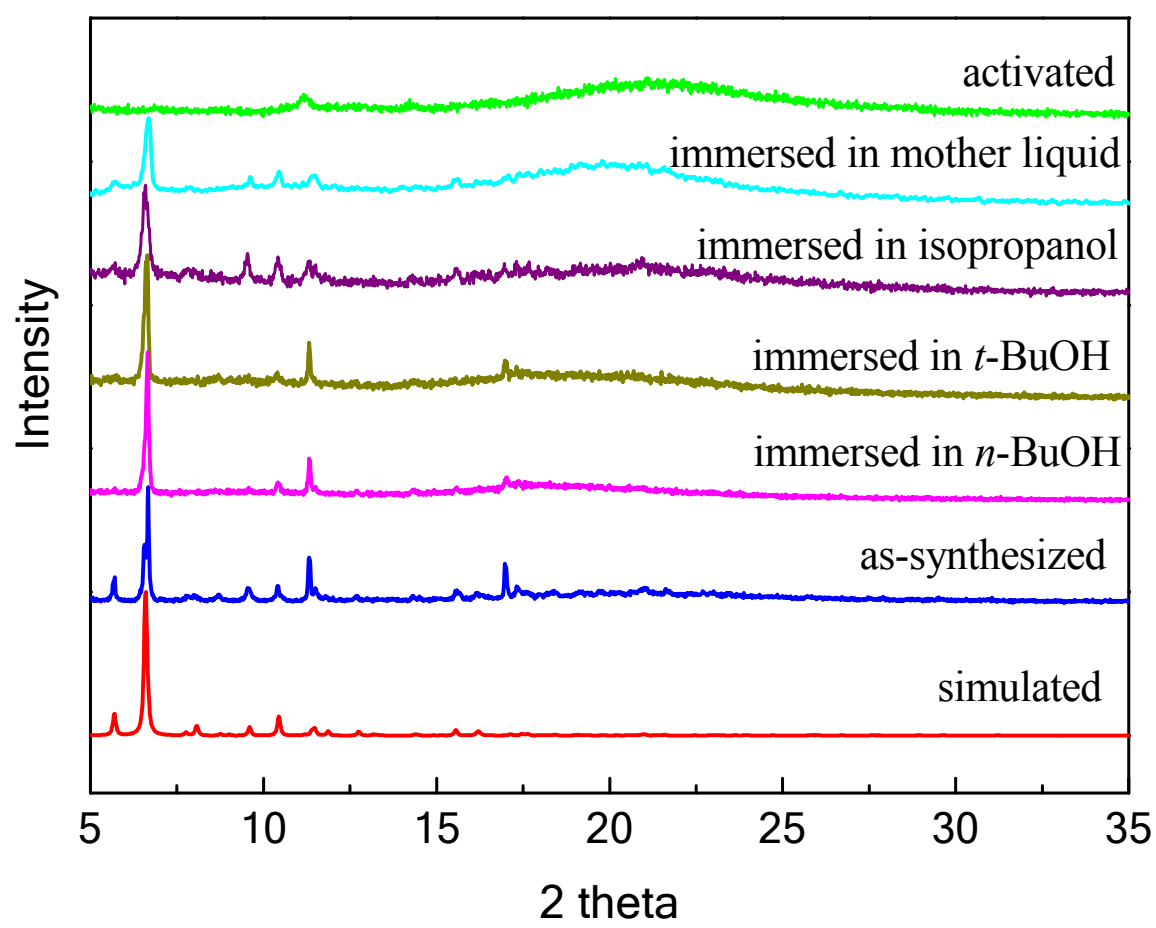

Figure S3. XRD patterns of VT-MOF-1 and other samples loaded with different solvent molecules. For the solvent loaded samples, the loss of the first peak might be caused by the loss of some crystallinity. The existence of amorphous hump in the sample immersed in mother liquid and activated sample should be caused by the amorphous state in the corresponding samples. 

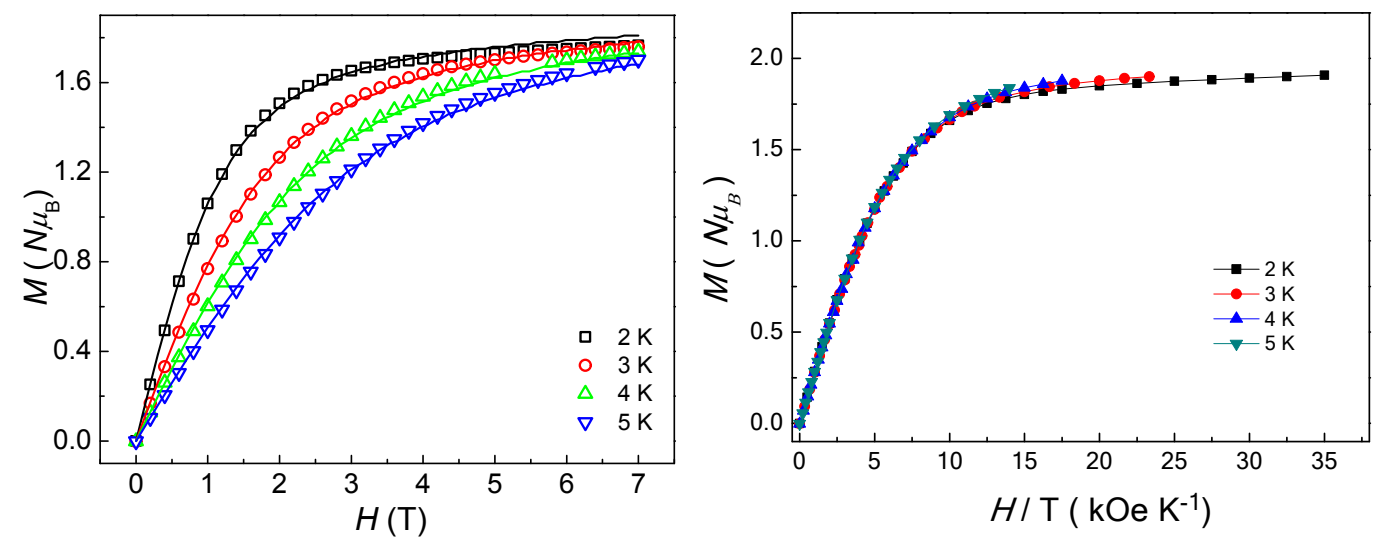

Figure S4. (left) Field dependence of the magnetization for the suspensions of VT-MOF-1 at 2, 3, 4, 5K. Solid red lines indicate the best fits with the PHI program. At $2 \mathrm{~K}$, the magnetization becomes 1.84 at $7 \mathrm{~T}$, smaller than $2.40 N \mu_{\mathrm{B}}$ for a cobalt(II) ion with $S=3 / 2$ caused by the the different population of the different Kramers levels, indicating the possibility of magnetic anisotropy in VT-MOF-1. (right) Experimental $M$ vs $H / \mathrm{T}$ plots at different temperatures of VT-MOF-1.

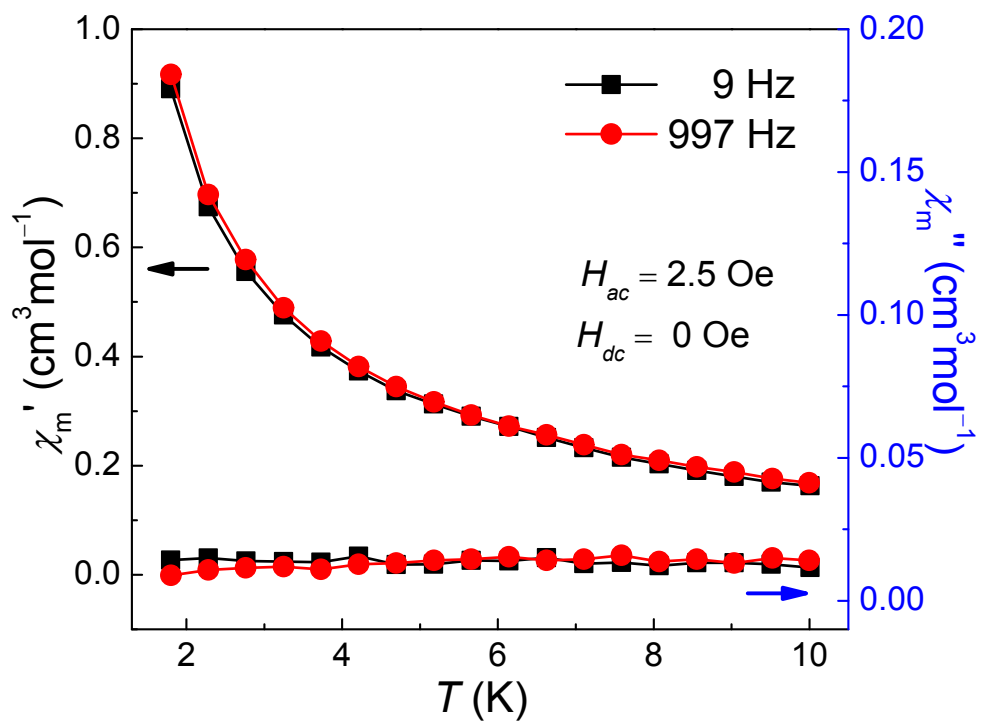

Figure S5. Temperature dependence of the in-phase $\left(\chi^{\prime}\right)$ and out-of-phase $\left(\chi^{\prime \prime}\right)$ ac magnetic susceptibility for complex VT-MOF-1 in zero dc field at 9 and $999 \mathrm{~Hz}$. 

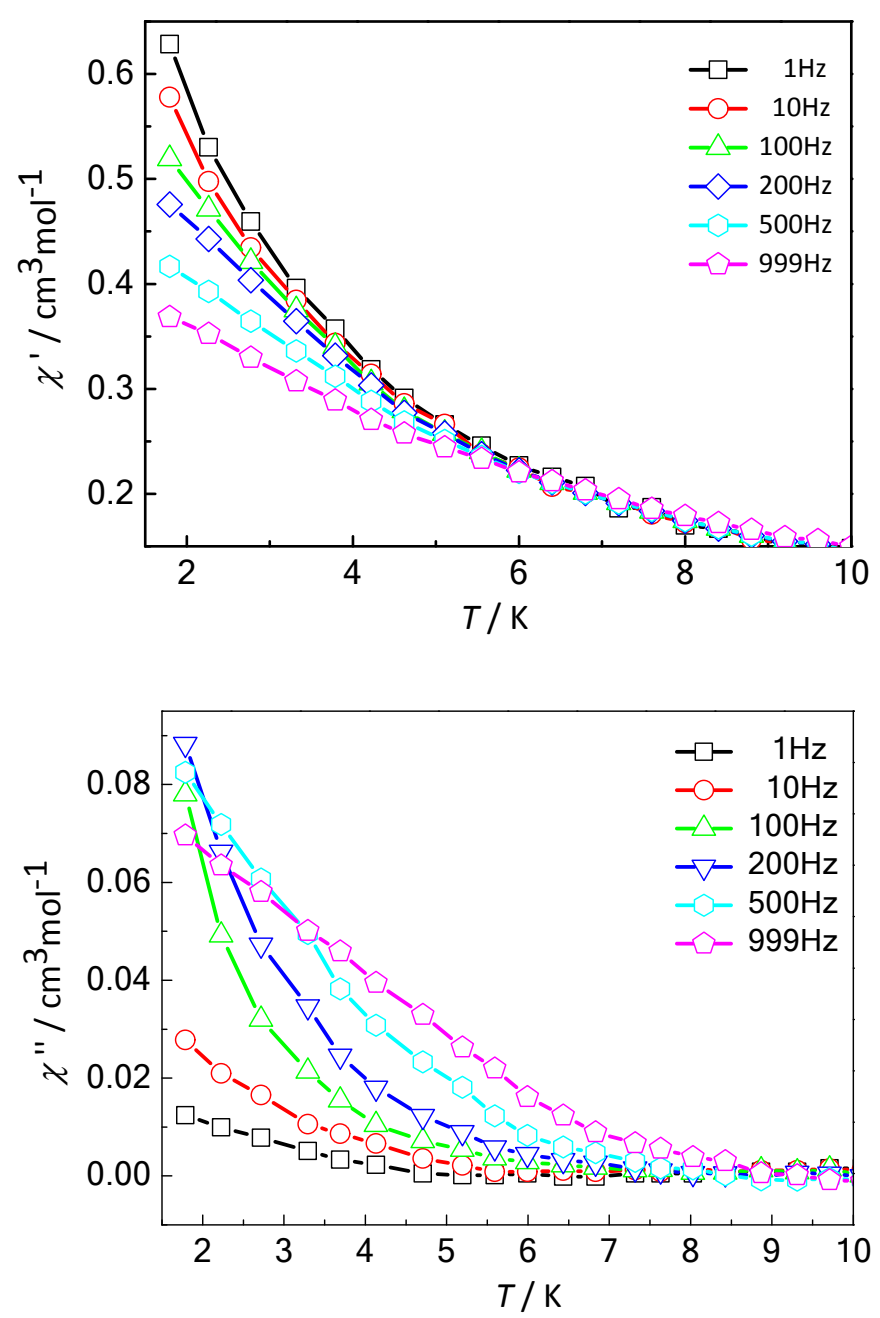

Figure S6. Temperature dependence of the in-phase $\left(\chi^{\prime}\right)$ and out-phase $\left(\chi^{\prime}\right)$ ac magnetic susceptibility for VT-MOF-1 from 1 to $999 \mathrm{~Hz}$ at 2000 Oe. The obvious peaks could not be detected due to the limitation measurement scope of equipment. The low temperature is $1.8 \mathrm{~K}$. The peak should be presented at the region lower thant $1.8 \mathrm{~K}$. 

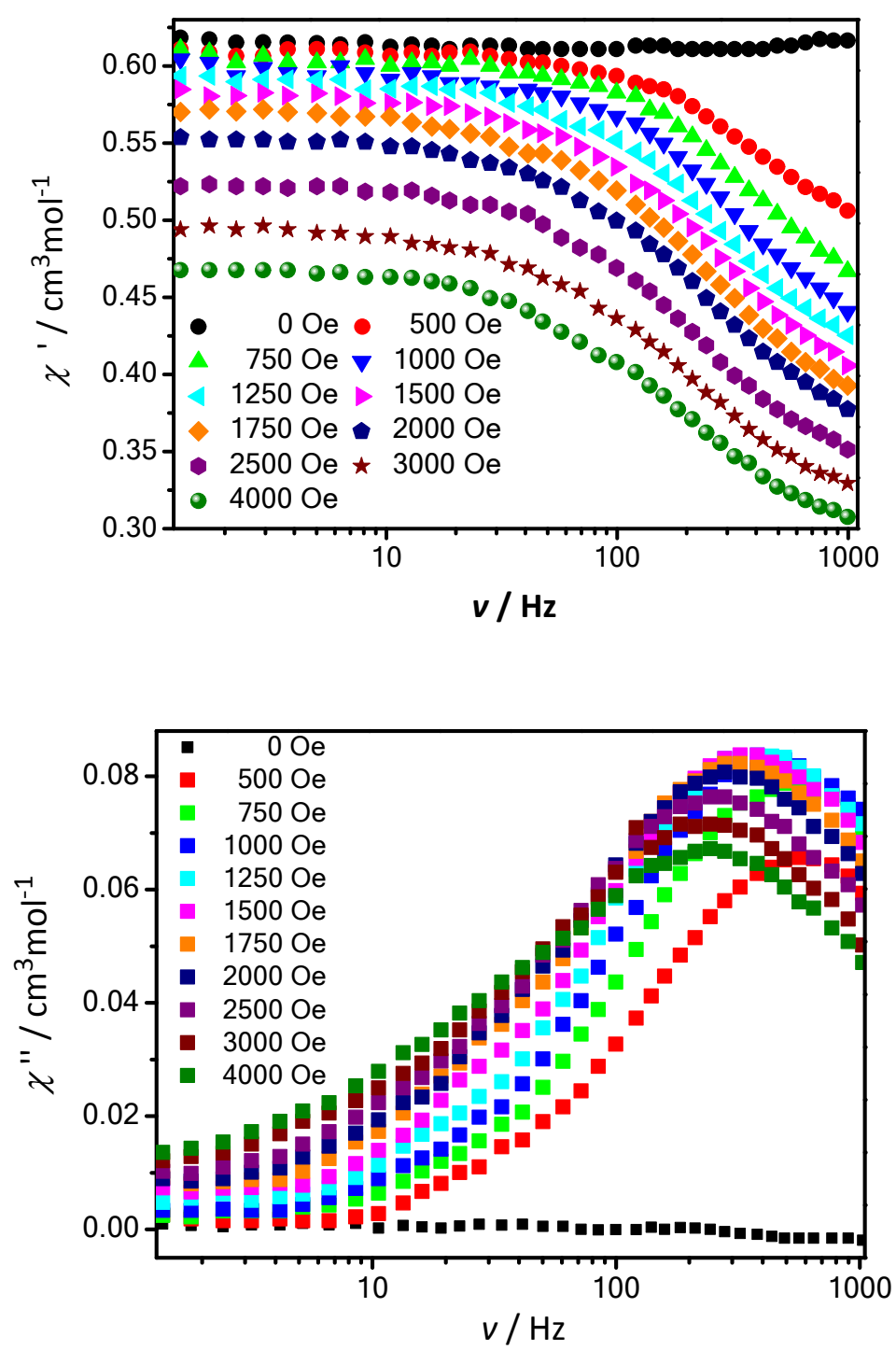

Figure S7. Frequency dependence of in-phase $\left(\chi^{\prime}\right)$ (up) and out-phase $\left(\chi^{\prime \prime}\right)$ (down) ac susceptibility at $2 \mathrm{~K}$ under various de fields for complex VT-MOF-1. 


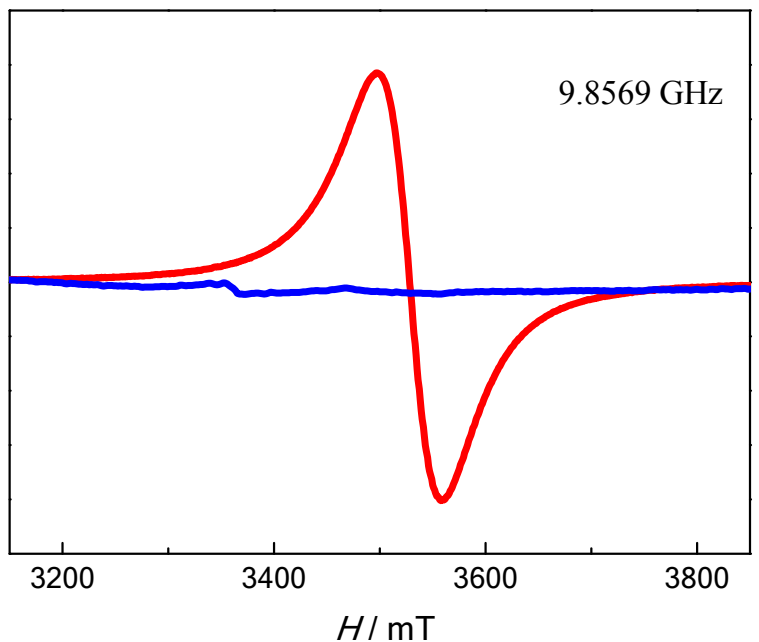

Figure S8. EPR spectrum of VT-MOF-1 at $100 \mathrm{~K}$ ( blue line ) and 293K ( red line ). The calculated g value equals to 2.01 .

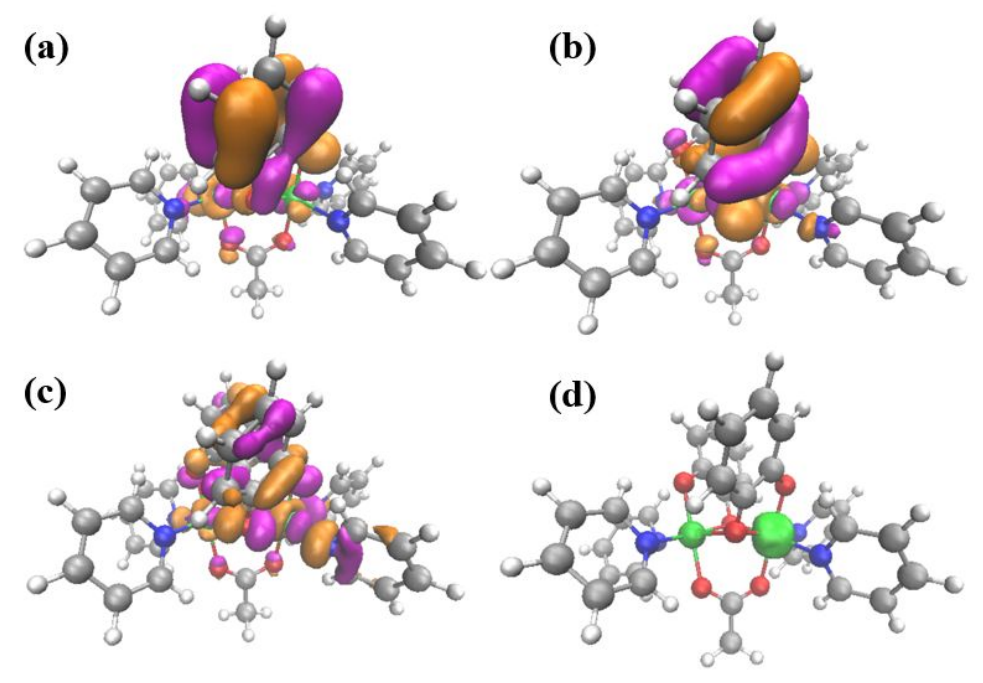

Figure S9. Frontier singly-occupied molecular orbitals (SOMOs) :174(a), 173(b),172(c) and spin density (d) of dinuclear cluster with spin state $S=3 / 2$ (Co1: left; Co2: right. density cuttoff $=0.030 \mathrm{au})$. 

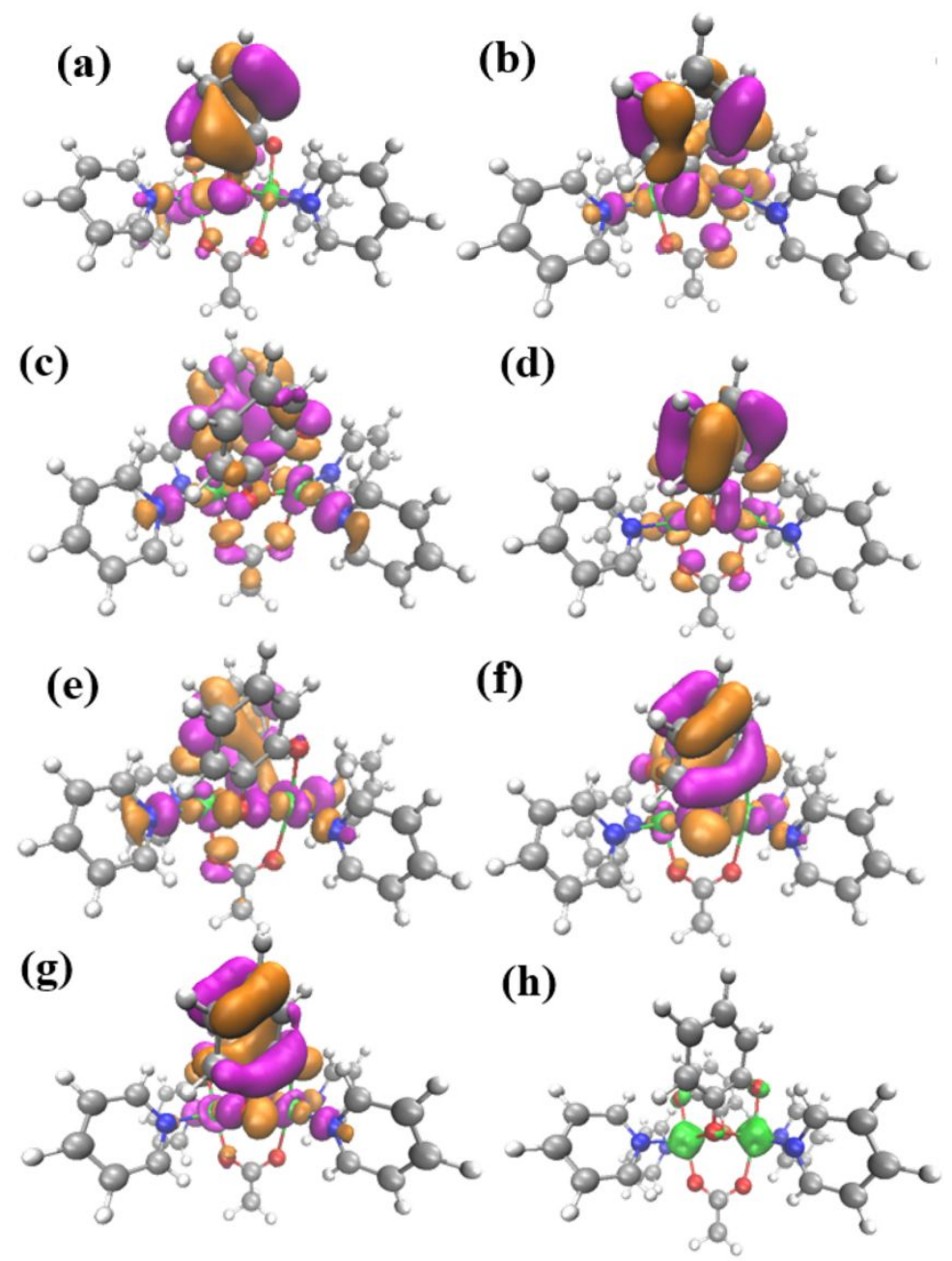

Figure S10. Frontier singly-occupied molecular orbitals (SOMOs) : 176(a),175(b), 174(c),173(d),172(e),171(f),170(g) and total spin density (h) of dinuclear cluster with spin state $S=2 \times(3 / 2)+1 / 2(\mathrm{Co} 1$ : left; Co2: right. density cuttoff $=0.030 \mathrm{au})$. 


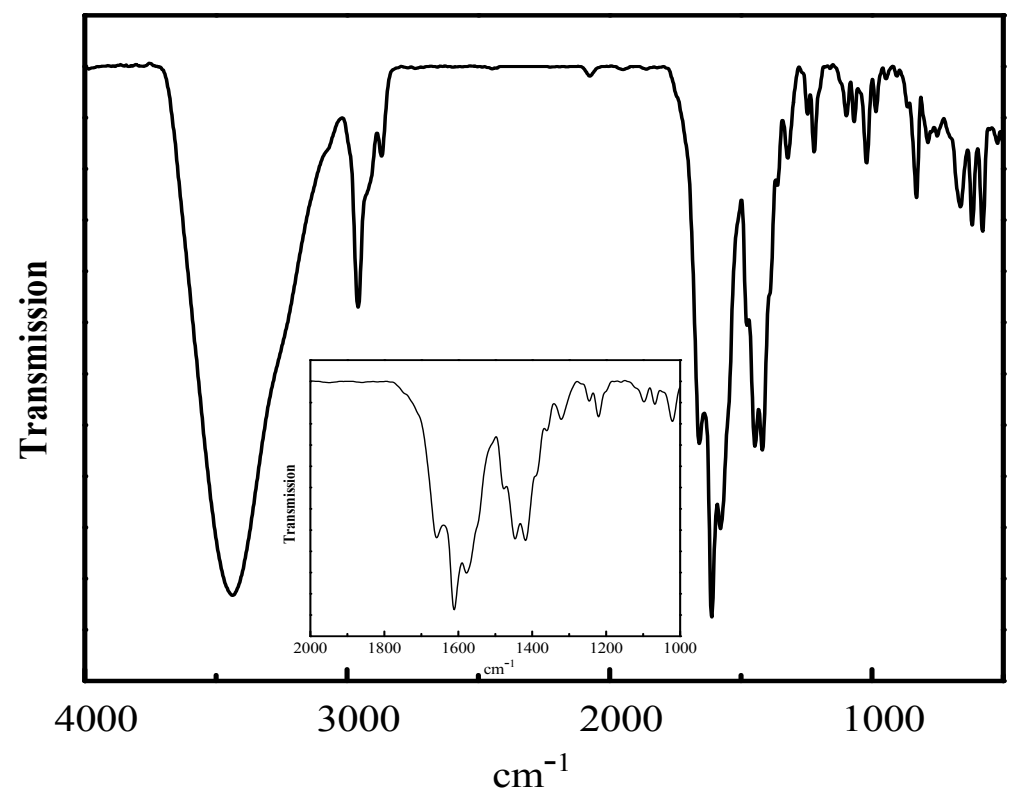

Figure S11. IR spectra of VT-MOF-1.

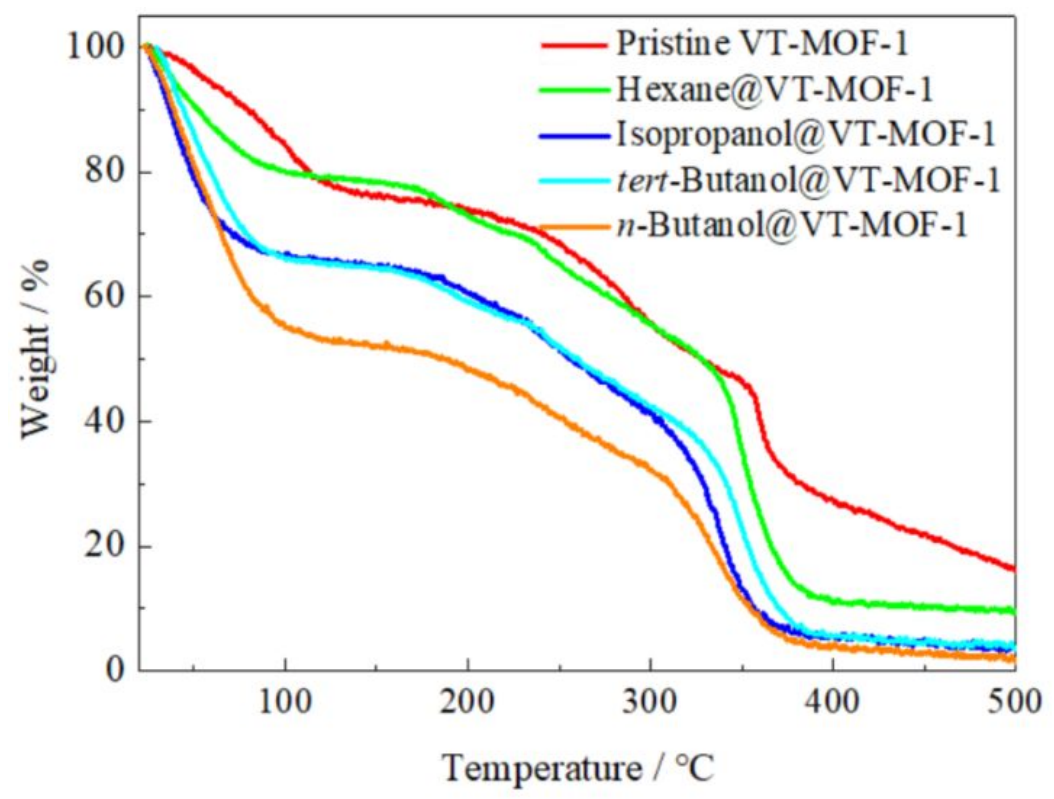

Figure S12. TGA curves of VT-MOF-1 and the sample with different solvents. With the order of increasing boil point as hexane, isopropanol, tert-butanol and $n$-butanol, the load amount of solvent molecules is increasing. For hexane@VT-MOF-1, $\left[\mathrm{Co}_{2}(\mathrm{OAc})(3,5-\mathrm{DBcat})_{2}(\mathrm{~L})\right] \cdot(\text { hexane })_{4} ; \quad$ For $\quad$ isopropanol@VT-MOF-1, $\left[\mathrm{Co}_{2}(\mathrm{OAc})(3,5-\mathrm{DBcat})_{2}(\mathrm{~L})\right] \cdot(\text { isopropanol })_{8} ; \quad$ For $\quad$ tert-butanol@VT-MOF-1, 
$\left[\mathrm{Co}_{2}(\mathrm{OAc})(3,5-\mathrm{DBcat})_{2}(\mathrm{~L})\right] \cdot(\text { tert-butanol })_{8} ; \quad$ For $\quad n$-butanol@VT-MOF-1, $\left[\mathrm{Co}_{2}(\mathrm{OAc})(3,5-\mathrm{DBcat})_{2}(\mathrm{~L})\right] \cdot(n \text {-butanol })_{12}$.

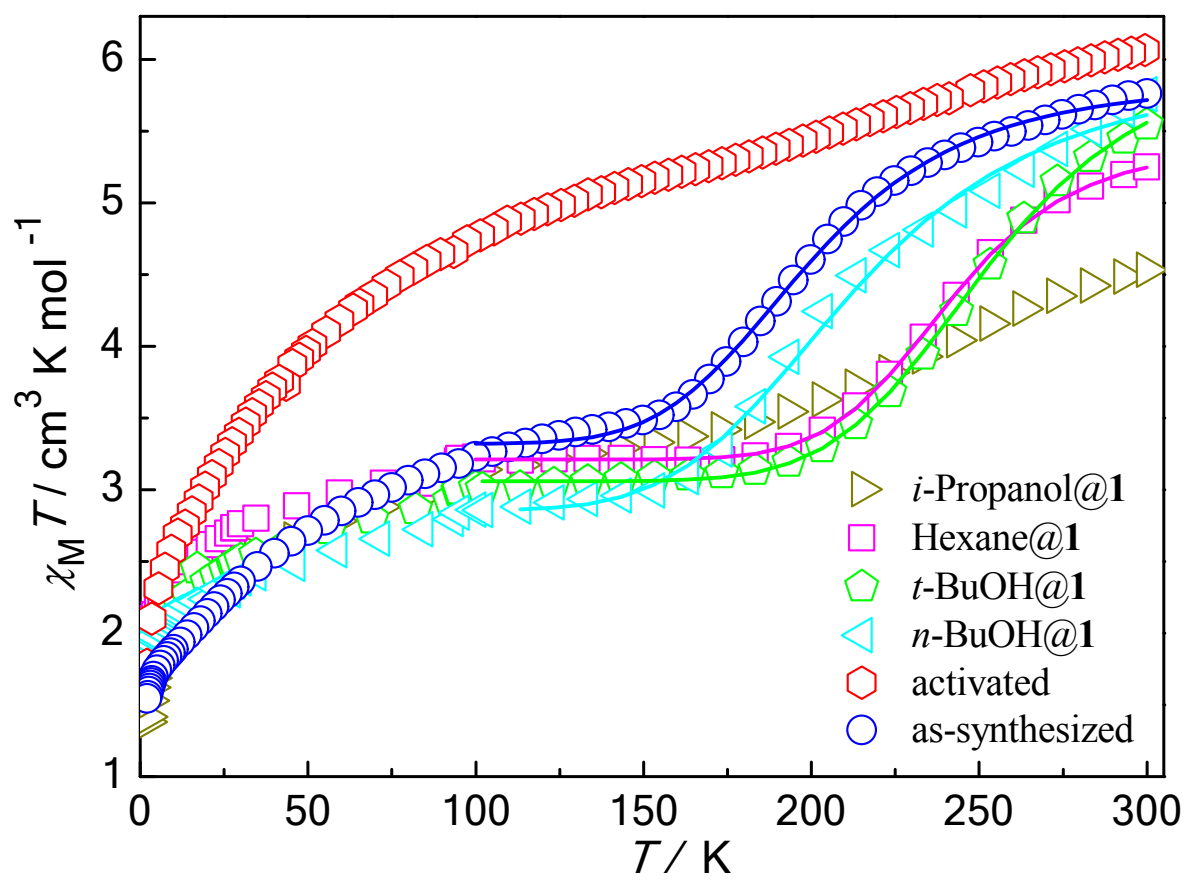

Figure S13. Variable temperature $\chi_{\mathrm{M}} T$ values vs $T$ plots ( dot: experiment; solid line: fitting from van's Hoff equation). The repeated transition behaviors of the original samples could be still detected after three times.The reversible temperature regions is 2-300 K. The irreversible VT behavior would be ascribed to the loss of the guest molecules at high temperature regions. No similar photo irradiation phenomenon at low temperature could be detected (Inorg. Chem. 50, 2011, 424).

\section{Fitting of Variable-temperature M-T curve}

In thermodynamic system, equilibrium constant ( $K_{\text {eq }}$ ) of the reaction equation ( see eq(3) is typically described as van's Hoff equation ${ }^{[6]}$ (see eq(4) ). For valence tautomerism $^{[8]}$, this equation of eq(4) can be converted into eq(5), involving the relation of five parameters: effective magnetic moment $\mu_{\mathrm{eff}}$, enthalpy change $\Delta \mathrm{H}$, entropy change $\Delta S$, high- and low-temperature limits of magnetic moment $\mu_{\text {eff }}(\mathrm{HT})$ 
and $\mu_{\text {eff }}(\mathrm{LT})$. Variable-temperature magnetic susceptibility data of complex 1 was fitted by van's Hoff equation. The obtained results is presented as table S7.

$$
\begin{aligned}
& {\left[\mathrm{Co}^{I I} \mathrm{Co}{ }^{\text {III }}(\mathrm{OAc})(3,5-\mathrm{DBcat})_{2}(\text { pyridine })_{4}\right] \stackrel{K_{e q}}{\rightleftharpoons}\left[\mathrm{Co}^{I I} \mathrm{Co}^{I I}(\mathrm{OAc})(3,5-\mathrm{DBcat})_{2}(\text { pyridine })_{4}\right]} \\
& \ln K_{e q}=-\frac{\Delta H}{R T}+\frac{\Delta S}{R} \\
& \mu_{e f f}=\sqrt{\frac{e^{\left(-\frac{\Delta H}{R T}+\frac{\Delta S}{R}\right)} \mu_{e f f}^{2}(H T)+\mu_{e f f}^{2}(L T)}{e^{\left(-\frac{\Delta H}{R T}+\frac{\Delta S}{R}\right)}+1}}
\end{aligned}
$$

Table S7. The fitted parameters from variable-temperature magnetic data of VT-MOF-1with different guest molecules by van's Hoff equation

\begin{tabular}{ccccc}
\hline & as-synthesize & $n$-BuOH@ & $t$-BuOH@1 & $n$-Hexane $@$ \\
& $\mathrm{~d}$ & 1 & & 1 \\
\hline$\Delta \mathrm{H}(\mathrm{kJ} / \mathrm{mol})$ & 13.71 & 12.58 & 21.51 & 25.31 \\
$\Delta \mathrm{S}(\mathrm{J} / \mathrm{mol})$ & 68.57 & 58.83 & 85.13 & 104.93 \\
$T_{1 / 2}(\mathrm{~K})^{*}$ & 199.95 & 213.76 & 252.63 & 241.22 \\
$\mu_{\mathrm{eff}}(\mathrm{HT})$ & 6.852 & 6.911 & 6.959 & 6.584 \\
$\mu_{\mathrm{eff}}(\mathrm{LT})$ & 5.153 & 4.780 & 4.946 & 5.067 \\
\hline
\end{tabular}

* The equilibrium temperature: $T_{1 / 2}$ is equal to the value of $\Delta H / \Delta S$.

Table S8. The parameters of different solvent in title MOF

\begin{tabular}{ccc}
\hline solvent & $\mathrm{S}_{\mathrm{m}}{ }_{\mathrm{m}}\left(\mathrm{g}, \mathrm{J} \mathrm{mol}^{-1} \mathrm{~K}^{-1}\right)$ & $T_{1 / 2}(\mathrm{~K})$ \\
\hline$n$-butanol & 361.59 & 213.76 \\
\hline Hexane & 388.85 & 241.22 \\
\hline
\end{tabular}




\begin{tabular}{ccc}
\hline tert-Butanol & 326.70 & 252.36 \\
\hline isopropanol & 309.20 & $>280 \mathrm{~K}$ \\
\hline
\end{tabular}

*The transition temperature for isopropanol is approximately calculated due to the incomplete transition in the whole observed temperature.

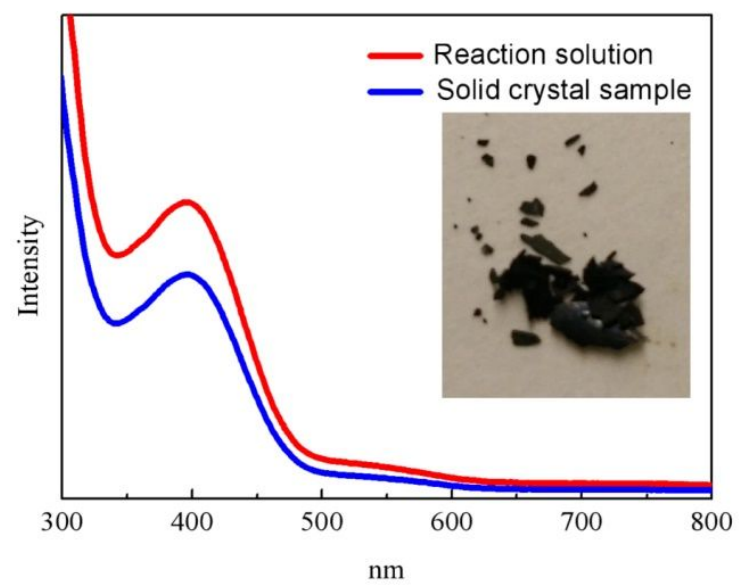

Figure S14. UV-Vis spectra of VT-MOF-1 in the reaction solution and solid state.

Table S9. Energy difference and relation formula between $J\left(J_{1}\right.$ and $\left.J_{2}\right)$ according to broken-symmetry method, which reveals that $\mathrm{Co}$ (II) ions and semiquinone radical features antiferromagnetic exchange $\left(-210.9 \mathrm{~cm}^{-1}\right)$, consistent with other reported results $^{9}$, while two $\mathrm{Co}$ (II) ions behaves very weak ferromagnetic coupling ( 47.78 $\mathrm{cm}^{-1}$ ) closely related to bond angle of Co-O-Co ( $98.5^{\circ}$ and $\left.93.5^{\circ}\right)$, similar to other Co-O-Co systems with very weak ferromagnetic coupling ${ }^{10}$.

\begin{tabular}{llll}
\hline Spin density & $\mathrm{S}_{\max }=2 \times(3 / 2)+1 / 2$ & $\mathrm{~S}=3 / 2$ & $\mathrm{~S}=1 / 2$ \\
\hline$\Delta \mathrm{E}\left(S-S_{\max }\right) / \mathrm{cm}^{-1}$ & 0 & -843.46 & -135.04 \\
Formula & - & $4 J_{1}$ & $6 J_{2}+2 J_{1}$ \\
\hline
\end{tabular}




\section{References}

[1] Gaussian 09, Revision A.1, M. J. Frisch, G. W. Trucks, H. B. Schlegel, G. E. Scuseria, M. A. Robb, J. R. Cheeseman, G. Scalmani, V. Barone, B. Mennucci, G. A. Petersson, H. Nakatsuji, M. Caricato, X. Li, H. P. Hratchian, A. F. Izmaylov, J. Bloino, G. Zheng, J. L. Sonnenberg, M. Hada, M. Ehara, K. Toyota, R. Fukuda, J. Hasegawa, M. Ishida, T. Nakajima, Y. Honda, O. Kitao, H. Nakai, T. Vreven, J. A. Montgomery, Jr., J. E. Peralta, F. Ogliaro, M. Bearpark, J. J. Heyd, E. Brothers, K. N. Kudin, V. N. Staroverov, R. Kobayashi, J. Normand, K. Raghavachari, A. Rendell, J. C. Burant, S. S. Iyengar, J. Tomasi, M. Cossi, N. Rega, J. M. Millam, M. Klene, J. E. Knox, J. B. Cross, V. Bakken, C. Adamo, J. Jaramillo, R. Gomperts, R. E. Stratmann, O. Yazyev, A. J. Austin, R. Cammi, C. Pomelli, J. W. Ochterski, R. L. Martin, K. Morokuma, V. G. Zakrzewski, G. A. Voth, P. Salvador, J. J. Dannenberg, S. Dapprich, A. D. Daniels, Ö. Farkas, J. B. Foresman, J. V. Ortiz, J. Cioslowski, and D. J. Fox, Gaussian, Inc.,Wallingford CT, 2009.

[2] M. Reiher, O. Salomon, B. A. Hess, Theor. Chem. Acc., 2001, 107, 48.

[3] D. Sato, Y. Shiota, G. Juhasz, K. Yoshizawa, J. Phys. Chem. A, 2010, 114, 12928.

[4] (a) L. Noodleman and J. G. N. Jr., J. Chem. Phys, 1979, 70, 4903; (b) L. Noodleman, J. Chem. Phys,1981, 74, 5737.

[5] J. A. Montgomery Jr., M. J. Frisch, J. W. Ochterski, and G. A. Petersson, J. Chem. Phys., 1999, 110, 2822-27.

[6] P. Atkins, Julio. De Paula, Physical Chemistry (8th ed.). 2016, W. H. Freeman and Company. p. 212. ISBN 0-7167-8759-8. 
[7] A. Bencini and F. Totti, J. Chem. Theory Comput., 2009, 5, 144.

[8] A. Witt, F. W. Heinemann, M. M. Khusniyarov, Chem. Sci., 2015, 6, 4599.

[9] V. I. Minkin, A. A. Starikova, A. G. Starikov, Dalton Trans., 2016, 45, 12103.

[10](a) M.-H. Zeng, M.-X. Yao, H. Liang, W.-X. Zhang, X.-M. Chen, Angew. Chem., Int. Ed. 2007, 46, 1832; (b) B. Li, Z. Li, R. Wei, F. Yu, X. Chen, Y. Xie, T. Zhang,J. Tao, Inorg. Chem. 2015, 54, 3331. 